\section{A role for $\beta$-actin in RNA polymerase III transcription}

\author{
Ping Hu, ${ }^{1} \mathrm{Si} \mathrm{Wu},{ }^{1}$ and Nouria Hernandez ${ }^{2,3}$ \\ ${ }^{1}$ Cold Spring Harbor Laboratory, Cold Spring Harbor, \\ New York 11724, USA; ${ }^{2}$ Howard Hughes Medical Institute, \\ Cold Spring Harbor Laboratory, Cold Spring Harbor, \\ New York 11724, USA
}

When transcription from the human U6 snRNA gene is reconstituted with recombinant factors and purified RNA polymerase III (pol III), pol III must be treated with CK2 to be active. We show that highly purified pol III contains associated $\beta$-actin, and $\beta$-actin localizes to an active U6 promoter in vivo. Pol III immunoprecipitated from IMR90 cells treated with a genotoxic agent lacks associated $\beta$-actin and is inactive in the reconstituted assay. Transcription is regained upon treatment of pol III with CK2 and addition of $\beta$-actin. This suggests that $\beta$-actin associated with pol III is essential for basal pol III transcription.

Supplemental material is available at http://www.genesdev.org.

Received August 27, 2004; revised version accepted October 8,2004

$\beta$-actin has been extensively studied as a cytoplasmic cytoskeletal protein that plays roles in intracellular trafficking as well as in driving changes in cellular shape associated with cell motility, cytokinesis, endocytosis, and cell adhesion (for reviews, see Brakebusch and Fassler 2003; Engqvist-Goldstein and Drubin 2003; Suetsugu and Takenawa 2003; Ascough 2004). However, $\beta$-actin is also a nuclear protein, and in this cellular compartment it is thought to play roles in the constitution of the nucleoskeleton, in RNA processing and export, and in transcription (for review, see Bettinger et al. 2004).

A role for actin in transcription is supported by two general lines of evidence. First, a number of experiments directly suggest an actin requirement for active transcription. Thus, a factor stimulating pol II transcription in vitro was found to copurify and probably correspond to actin (Egly et al. 1984). Further, injection of anti-actin antibodies into the nuclei of salamander oocytes results in a contraction of the lateral loops of lampbrush chromosomes similar to that observed upon injection of transcription inhibitors, consistent with a role in transcription (Scheer et al. 1984). Injection of a peptide acting as a competitive inhibitor of a protein-protein contact involving actin and the heterogenous nuclear RNP (hnRNP) protein hrp36 into the salivary glands of Chironomus tentans disrupts global RNA polymerase (pol) II transcription as measured by bromo-UTP incorporation,

[Keywords: RNA polymerase III; $\beta$-actin; human U6 snRNA promoter; CK2]

${ }^{3}$ Corresponding author.

E-MAIL Hernande@cshl.edu; FAX (516) 367-6801.

Article published online ahead of print. Article and publication date are at http://www.genesdev.org/cgi/doi/10.1101/gad.1250804. an effect that is due at least in part to a decrease in elongation as measured by run-on assays (Percipalle et al. 2003). Moreover, actin and nuclear myosin (NM1) associate with RNA polymerase I, and NM1 can be localized to active nucleolar transcription sites, an observation that has prompted the suggestion that actin and NM1 may serve as a nucleolar motor involved in the transcription of ribosomal RNA genes (Fomproix and Percipalle 2004). Second, at least some of the nuclear $\beta$-actin is an integral component of chromatin remodeling complexes such as the BAF, BAP, and INO80 complexes, as well as histone acetyl transferase complexes such as the Nu4A and TIP60 complexes (for review, see Bettinger et al. 2004). In the SWI/SNF-like BAF complex, $\beta$-actin stimulates the ATPase activity of the Brgl subunit and is required for association of the complex with the nuclear matrix (Zhao et al. 1998). Moreover, it can, at least in vitro, mediate actin filament binding of the complex in a phosphatidylinositol 4,5-biphosphate (PIP2)-dependent manner (Rando et al. 2002). In the INO80 complex, actin may be required for efficient DNA binding, ATPase activity, and nucleosome mobilization, as INO80 complexes lacking actin as well as the actin-related proteins Arp4 and Arp8 are deficient for these activities (Shen et al. 2003).

We recently purified pol III from a human cell line expressing a doubly tagged pol III subunit and observed that a number of polypeptides, one of them $\beta$-actin, copurify with the enzyme. We show here that $\beta$-actin is stably associated with pol III and is required for pol III transcription in a reconstituted in vitro transcription system. This suggests that $\beta$-actin plays a direct role in transcription, independent of any nuclear localization function.

\section{Results and Discussion}

\section{$\beta$-actin is tightly associated with pol III}

In earlier reports, we described the extensive purification of a tagged pol III complex from a Hela cell line expressing Flag- and His-tagged HsRPC4 (HsRPC53) through ammonium sulfate precipitation followed by anti-Flag immunoaffinity chromatography, nickel affinity chromatography, and mini-Q chromatography, as summarized in Figure 1A (Hu et al. 2002, 2003). In this purification protocol, we observed copurification of pol III subunits with $\beta$-actin (Hu et al. 2003), as shown in Figure 1B for RPC1, the largest pol III subunit. Intriguingly, in this purification, the U6 transcription activity profile across the fractions closely followed the $\beta$-actin profile. In particular, fraction 12, which contains less RPC1 but more $\beta$-actin than fraction 10 , was more active for U6 transcription than fraction 10 (see Fig. 4 in $\mathrm{Hu}$ et al. 2003), consistent with a possible role of $\beta$-actin in pol III transcription. To determine whether $\beta$-actin might also copurify with endogenous, untagged pol III, we partially purified the endogenous enzyme according to a protocol we developed previously (Sepehri Chong et al. 2001). At the last step of this purification scheme, which as summarized in Figure 1C corresponds to a sucrose gradient, pol III formed a single peak and could reconstitute U6 transcription when supplemented with recombinant TBP, Brf2, Bdp1, and SNAP (Sepehri Chong et al. 2001). 
A

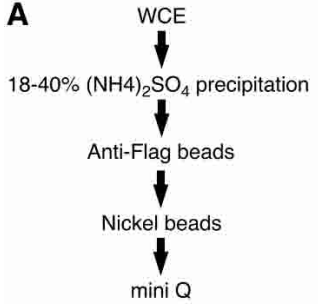

C

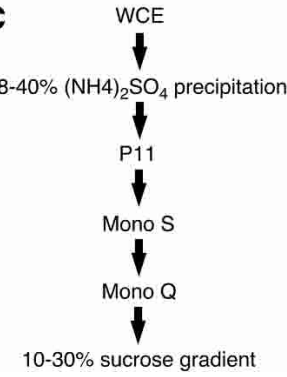

B

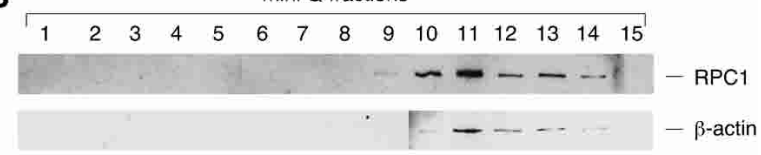

D

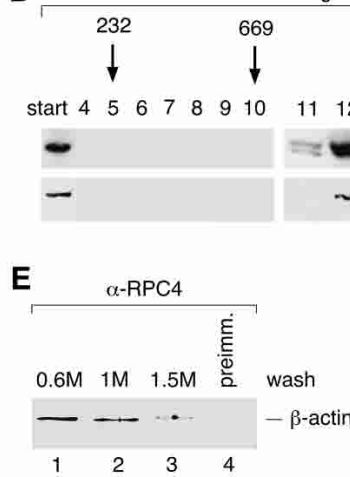

crose gradient fractions
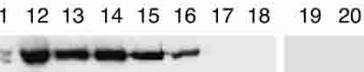

$-$

$\mathbf{F}$

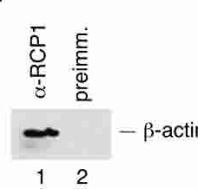

Figure 1. $\beta$-actin is associated with pol III. $(A)$ Purification scheme of tagged pol III from a HeLa cell line expressing Flag- and His-tagged RPC4. (B) $\beta$-actin and RPC1 copurify during mono Q chromatography. This panel is reprinted from Figure 4D in Hu et al. (2003) (C) 2003 with permission from Elsevier) and shows immunoblots performed with anti-RPCl and anti$\beta$-actin antibodies. (C) Purification scheme of endogenous pol III. (D) $\beta$-actin and pol III copurify during sucrose gradient fractionation. The immunoblot was probed as in $B$. (E) The association of $\beta$-actin with pol III is resistant to high salt. HeLa whole-cell extracts were incubated with anti-RPC4 beads, the beads were then washed with $0.6,1$, or $1.5 \mathrm{M} \mathrm{KCl}$ as indicated above the lanes, and the bound material was eluted, fractionated on a gel, and immunoblotted with an anti- $\beta$-actin antibody. In lane 4 , preimmune antibody beads were used. $(F)$ HeLa whole-cell extract was incubated with anti-RPC1 beads, the beads were washed with $0.6 \mathrm{M}$ $\mathrm{KCl}$, and the bound material was analyzed as in $E .(G)$ As in $F$ except that the HeLa whole-cell extract was incubated with anti-RPC5 beads.

We examined the distribution of $\beta$-actin across the sucrose gradient fractions by immunoblot. We observed a single peak of $\beta$-actin, and as shown in Figure 1D, this peak coincided with the peak of RPC1. Thus, upon purification of both tagged and endogenous pol III complexes, we observe copurification of $\beta$-actin, suggesting that $\beta$-actin is associated with pol III.

To examine how tightly $\beta$-actin associates with pol III, we performed nondenaturing immunoprecipitations from HeLa cell extracts with an anti-RPC4 antibody. The immunoprecipitates were then extensively washed with buffers containing $600 \mathrm{mM} \mathrm{KCl}, 1 \mathrm{M} \mathrm{KCl}$, and $1.5 \mathrm{M}$ $\mathrm{KCl}$. As shown in Figure 1E, in all cases $\beta$-actin was specifically detected in the material immunoprecipitated with the anti-RPC4, but not with preimmune, antibody, although the signal was weaker in the sample washed at the highest salt concentration (Fig. 1E, cf. lanes 3 and 1). We could also detect $\beta$-actin in immunoprecipitates (washed with $600 \mathrm{mM} \mathrm{KCl}$ ) performed with anti-RPC1 and anti-RPC5, but not the corresponding preimmune, antibodies, as shown in Figure 1F and G. In contrast, the transcription factor Oct-1 was not detected in these immunoprecipitates (data not shown).

To determine whether $\beta$-actin can directly associate with individual pol III subunits, we expressed 15 of the 17 pol III subunits as HA-tagged polypeptides by translation in vitro, as well as untagged $\beta$-actin, and tested them in coimmunoprecipitation experiments. Out of all the pol III subunits except for RPABC1 (RPB5) and RPABC5 (RPB10), which were not tested, we found that $\beta$-actin can associate directly with RPC3 (RPC62), RPABC2 (RPB6), and RPABC3 (RPB8) (see Supplemen-

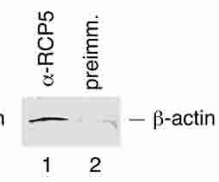

tary Table S1). Together, the results described thus far suggest that $\beta$-actin is associated very tightly with the pol III complex, probably through direct protein-protein interactions with one of more of the RPC3, RPABC2, and RPABC3 subunits.

Both $\mathrm{RPABC} 2$ and $\mathrm{RPABC} 3$ are common to all three RNA polymerases (for review, see Schramm and Hernandez 2002). Strikingly, in the crystal structure of pol II, they are located next to each other at the surface of the enzyme (Cramer et al. 2001), raising the possibility that they form a patch, most likely in the three RNA polymerases, for $\beta$-actin attachment. Indeed, actin has also been reported to associate with pol I (Fomproix and Percipalle 2004). RPC3 is unique to pol III and so we cannot infer its location in the pol III enzyme from the pol II crystal structure. This subunit is part of a subcomplex that also contains two other subunits unique to pol III, RPC6 (RPC39) and RPC7 (RPC32) (Wang and Roeder 1997), and in the yeast enzyme, photochemical cross-linking experiments performed with a transcription initiation complex indicated that it makes contact with the DNA (Bartholomew et al. 1993). Perhaps this subunit resides next to RPABC2 and RPABC3 in the three-dimensional structure of pol III. Alternatively, it may constitute a separate $\beta$-actin anchor site on the pol III enzyme.

\section{$\beta$-actin is located at promoter sequences of an actively transcribed U6 gene in vivo}

Given the tight association of $\beta$-actin with pol III, we tested whether $\beta$-actin is associated with an actively transcribed U6 gene in vivo by chromatin immunoprecipitation assays as described (Schramm et al. 2000; Zhao et al. 2001; Hu et al. 2003). The chromatin immunoprecipitations were performed in actively growing Hela and IMR90 cells, with similar results. As shown in Figure 2, both the control primers (labeled C), which hybridize to the promoter region of the transcriptionally inactive U6-4 gene, and the test primers (labeled T), which hybridize to the promoter region of the actively transcribed U6-1 gene (Domitrovich and Kunkel 2003), amplified the expected fragments from the starting chromatin material (Fig. 2, lanes 2,3), but not from material mock-immunoprecipitated without antibody (Fig. 2, lanes 4,5$)$. When we performed the immunoprecipitation with an antibody against the largest subunit of $\mathrm{SNAP}_{\mathrm{c}}$ (SNAP190), which is required for U6 transcription, the promoter region of the actively transcribed U6 gene, but not that of the transcriptionally inactive U6 gene, was amplified specifically, as expected (Fig. 2, lanes 6,7). In contrast, with an antibody against TFIIB, which is not required for U6 transcription, neither the promoter region of the active U6 gene nor that of the inactive U6 gene was amplified, as expected (Fig. 2, lanes 8,9). Thus, 
Hu et al.

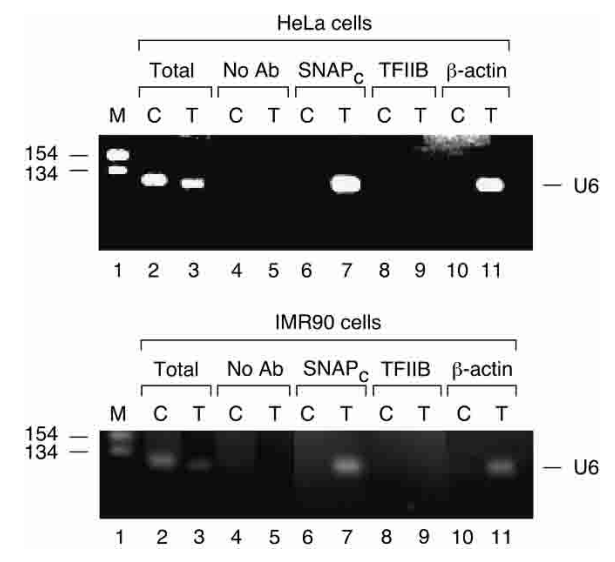

Figure 2. $\beta$-actin can be localized to the promoter sequences of an actively transcribed U6 gene. Cross-linked and sonicated chromatin from actively growing Hela (top panel) or IMR90 (bottom panel) cells was incubated with either no antibody (lanes 4,5), or antibodies against the SNAP190 subunit of SNAP $($ CS696, lanes 6,7), TFIIB (CS396, lanes 8,9), or $\beta$-actin (AC-74, Sigma, lanes 10,11). In each case, the immunoprecipitated material was analyzed by PCR with test primers $(\mathrm{T})$ specific for the promoter of an actively transcribed U6 gene, and control primers (C) specific for the promoter of an inactive U6 gene. In lanes 2 and 3, the starting chromatin material was directly analyzed by PCR. The order of the lanes in the bottom panel, which are from the same gel, has been rearranged for clarity.

the chromatin immunoprecipitations are highly specific. Very strikingly, with an antibody directed against $\beta$-actin, the promoter region of the actively transcribed U6 gene was specifically amplified (Fig. 2, lanes 10,11). Thus, in vivo, $\beta$-actin is located close to the promoter region of an actively transcribed U6 gene, consistent with the possibility that it participates in the transcription process.

\section{$\beta$-actin dissociates from pol III upon MMS treatment}

To explore a possible role for $\beta$-actin in pol III transcription, we searched for conditions under which $\beta$-actin might be dissociated from the pol III complex, such that we might compare the transcription activities of pol III with or without $\beta$-actin. Methane methylsulfonate (MMS) treatment induces DNA damage and is known to repress pol III transcription (Crighton et al. 2003). We treated IMR90 cells with MMS and checked p53 levels by immunoblot at various time points after treatment. As shown in Figure 3A and quantitated (after normalization to the TFIIB internal control signal) in Figure 3B, p53 levels peaked $2 \mathrm{~h}$ after MMS treatment and then diminished slowly over time, remaining detectable $5 \mathrm{~h}$ after MMS treatment. This suggested that the cell response to MMS peaks $2 \mathrm{~h}$ after treatment, and we therefore chose this time point to perform immunoprecipitations with an anti-RPC4 antibody. The immunoprecipitated material was then probed with an anti- $\beta$-actin antibody.

As shown in Figure 3C, neither the RPC4 antibody beads alone (Fig. 3C, lane 1) nor material precipitated with pre-immune antibody beads (Fig. 3C, lanes 2,3) contained detectable amounts of $\beta$-actin. When the immunoprecipitations were performed with extracts from actively growing IMR90 cells, the RPC1 pol III subunit as well $\beta$-actin were coimmunoprecipitated with RPC4 (Fig. 3C, lane 4), consistent with the results above in
HeLa cells (Fig. 1E). However, with extracts from MMStreated IMR90 cells, no $\beta$-actin was detected in the antiRPC4 immunoprecipitate, even though RPC1 was coimmunoprecipitated as efficiently as in the actively growing IMR90 cells (Fig. 3C, cf. lanes 4 and 5). Yet, similar levels of $\beta$-actin (as well as RPC1) were present in the starting extracts (Fig. 3C, lanes 6,7), indicating that MMS treatment does not significantly change the total levels of $\beta$-actin. Together, these results suggest that after DNA damage agent treatment, $\beta$-actin is dissociated from the pol III complex in IMR90 cells. Interestingly, this effect was much less pronounced in HeLa cells, where only a low proportion of the pol III-associated $\beta$-actin dissociated from the enzyme upon treatment with MMS (data not shown).

The dissociation of $\beta$-actin from pol III might be the result of a global disruption or distortion of cytoskeletal structures. We therefore examined the $\alpha$-tubulin and ac-

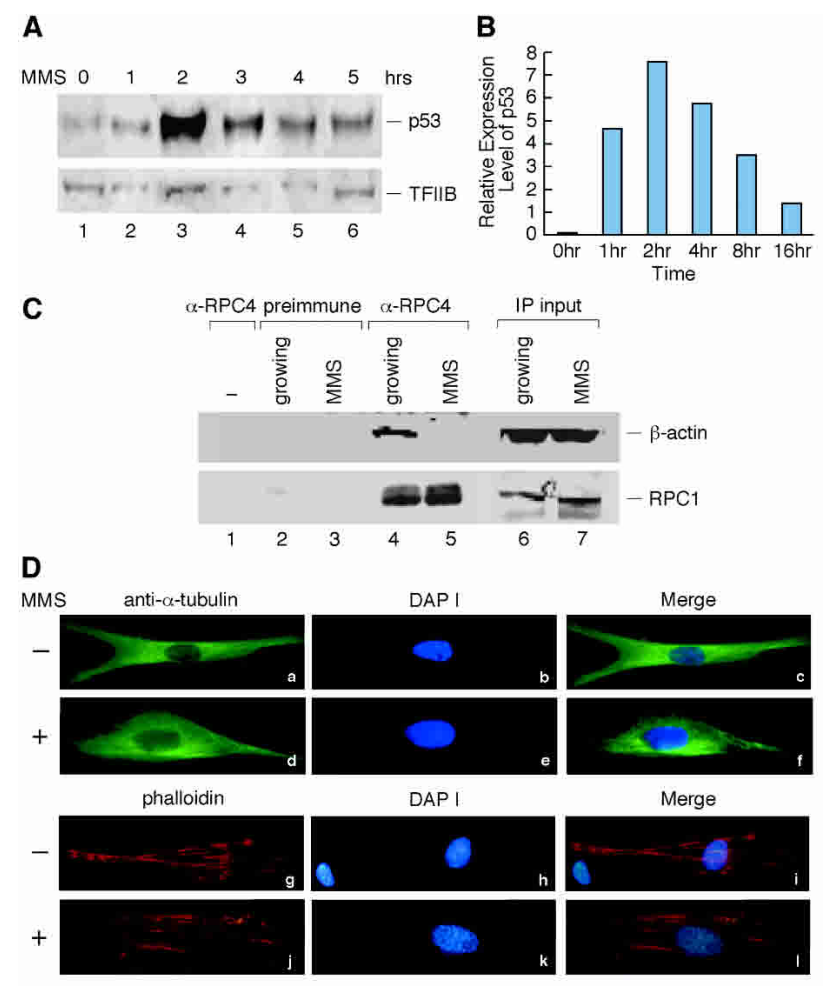

Figure 3. $\beta$-actin dissociates from pol III upon MMS treatment in IMR90 cells. $(A)$ p53 peaks $2 \mathrm{~h}$ after MMS treatment. IMR90 cells were treated with $1 \mathrm{mM}$ MMS. Samples were analyzed by immunoblotting with anti-p53 and anti-TFIIB antibodies at the times indicated after MMS treatment. $(B)$ Quantitation of the p53 signals in $A$ after normalization to the TFIIB internal control. $(C) \beta$-actin dissociates from pol III after MMS treatment. Extracts from either actively growing (lanes 2,4) or MMS-treated (lanes 3,5) IMR90 cells were incubated with preimmune (lanes 2,3) or anti-RPC4 (lanes 4,5) antibody beads, the beads were washed with $0.6 \mathrm{M} \mathrm{KCl}$, and the bound material was eluted and analyzed by immunoblotting with anti- $\beta$-actin and anti-RPC1 antibodies. In lane 1 , the anti-RPC4 beads were not incubated with extract prior to immunoblotting analysis of the bound material. Lanes 6 and 7 show immunoblots of the starting material that was used for the immunoprecipitations. (D) MMS treatment does not change the overall cytoskeleton structure. Actively growing (panels $a-c, g-i$ ) and MMS-treated (panels $d-f, j-1)$ IMR90 cells were stained with DAPI and either an immunofluorescent-labeled anti- $\beta$-tubulin antibody or Alexa Fluor 568labeled phalloidin, which stains filamentous actin. 
tin networks by immunofluorescence with an anti- $\alpha$-tubulin antibody and with phalloidin, which binds to fibrillar actin, with and without MMS treatment. Figure 3D shows examples of cells either left untreated or treated with MMS. We could not detect any global change in the overall tubulin or fibrillar actin networks after MMS treatment (Fig. 3D, cf. panels a-c and d-f, and panels $g-i$ and $j-1)$, suggesting that the dissociation of $\beta$-actin from pol III is not the result of an overall disruption of the cytoskeletal structure.

\section{The U6 initiation complex is disrupted after MMS treatment}

We carried out chromatin immunoprecipitations with quantitative PCR (qPCR) to monitor any changes on U6-1 promoter occupancy prior to, and after, MMS treatment. Figure 4A shows the ratios of the signals obtained in material immunoprecipitated from either actively growing or MMS-treated IMR90 cells to the signals obtained in material immunoprecipitated from MMStreated cells. Thus, for MMS-treated cells, the ratio is always 1. For RNA polymerase III, there was a twofold decrease in the amount of protein associated with the U6-1 promoter after MMS treatment. For TBP and Bdp1, the reduction was larger, seven- and fourfold, respectively. Strikingly, the levels of $\beta$-actin associated with the U6-1 promoter region decreased more than sevenfold after MMS treatment. These results suggest that upon MMS treatment, the U6 initiation complex as well as $\beta$-actin are largely dissociated from promoter sequences. Notably, there is a much larger decrease in $\beta$-actin association with the U6 promoter region compared to dissociation of pol III, consistent with the idea that $\beta$-actin dissociates from pol III, but that only some of the pol III dissociates from U6 sequences.

\section{$\beta$-actin is required for U6 transcription}

We prepared whole-cell extracts from logarithmically growing and MMS-treated IMR90 cells and tested their abilities to support pol III transcription. As shown in Figure 4B, extracts from untreated IMR90 cells supported robust transcription from both the type 3 U6 and the type 2 VAI promoters (Fig. 4B, lane 1), whereas extracts from MMS-treated cells showed less activity, even over a wide titration of extract (Fig. 4B, cf. lanes 2-5 and 1), consistent with the inhibition of tRNA transcription in extracts from MMS-treated HeLa cells observed by others (Crighton et al. 2003). To determine whether pol III was active in extracts from MMS-treated cells, we took advantage of a minimal U6 transcription system we developed previously (Hu et al. 2003). We immunoprecipitated pol III from either untreated or MMS-treated IMR90 cells with an anti-RPC4 antibody and checked that equal amounts of enzyme were precipitated in both cases by immunoblotting with an anti-RPC1 antibody, as shown in Figure 4C. We then supplemented these pol III complexes with recombinant TBP, Brf2, Bdp1, and $\mathrm{SNAP}_{\mathrm{c}}$, thus eliminating any potential MMS effect on these proteins and allowing a direct comparison of the activities of the enzymes. As shown in Figure 4D, pol III immunoprecipitated from untreated IMR90 cells supported robust transcription in the minimal system (Fig. 4D, lane 1), whereas pol III from MMS-treated cells was inactive (Fig. 4D, lanes 2,3). Pol III repression of type 2 tRNA promoters after MMS treatment results at least in

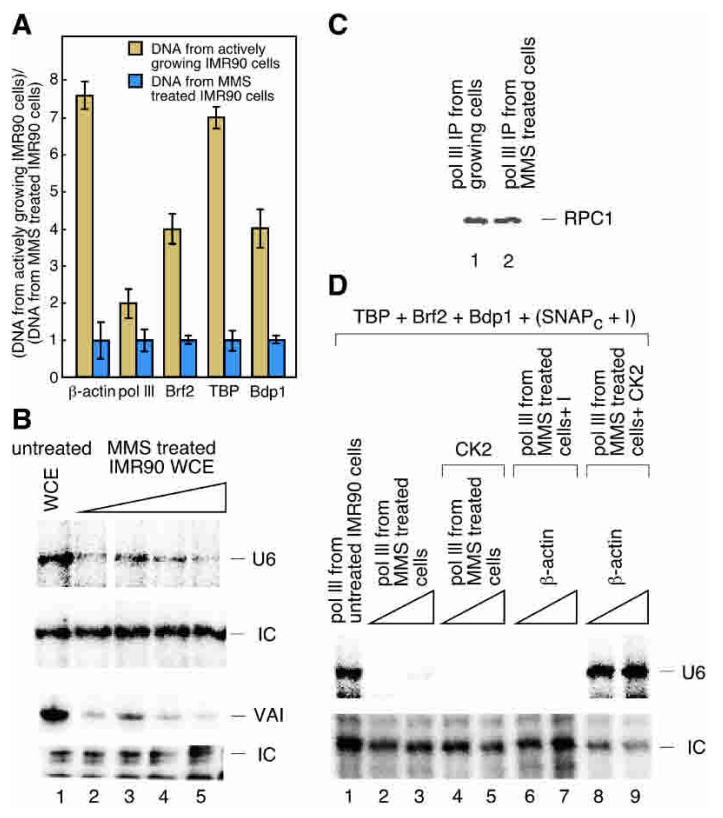

Figure 4. $\beta$-actin is required for U6 transcription in vitro. $(A) \beta$-actin dissociates from an active U6 promoter upon MMS treatment. The $Y$-axis indicates the ratio of DNA amplified from actively growing IMR90 cells to DNA amplified from MMS-treated IMR90 cells. The error bars are based on three independent PCR reactions with the same templates and primers. $(B)$ Extracts from MMS-treated IMR90 cells show reduced pol III transcription. Extracts from actively growing (lane 1) and MMS-treated (lanes 2-5) IMR90 cells were tested for transcription from the U6 and VAI promoters. (IC) Internal control for RNA recovery. $(C)$ Equal amounts of pol III were precipitated from untreated and IMR90-treated cells. Pol III was immunoprecipitated with an anti-RPC4 antibody, and the bound material was analyzed by immunoblotting with anti-RPC1 antibodies. (D) $\beta$-actin is required for U6 transcription in a reconstituted transcription system. SNAP $_{c}$ purified from baculovirus-infected insect cells was treated with $60 \mu \mathrm{M}$ LY294002 followed by $5 \mathrm{U}$ of CK2 holoenzyme $\left(\mathrm{SNAP}_{\mathrm{c}}+\mathrm{I}\right)$. TBP, Brf2, and Bdp1 were purified from $E$. coli. These factors were supplemented with $6 \mu \mathrm{L}$ of pol III immunoprecipitated from actively growing (lane 1) or $6 \mu \mathrm{L}$ (lanes 2,4,6-9) and $12 \mu \mathrm{L}$ (lanes 3,5) of pol III immunoprecipitated from MMStreated IMR90 cells. In lanes 4, 5, 8, and 9, immunoprecipitated pol III was incubated with $5 \mathrm{U}$ of CK2 holoenzyme for $10 \mathrm{~min}$ at $30^{\circ} \mathrm{C}$ followed by addition of $60 \mu \mathrm{M}$ LY294002 (CK2). In lanes 6 and 7, immunoprecipitated pol III was incubated first with LY294002 and then with CK2. In lanes 6-9, increasing amounts (50 and $100 \mathrm{ng}$ ) of $\beta$-actin were added to the reactions. The various combinations of factors were then tested for U6 transcription.

part from p53-mediated effects on TFIIIB; the TFIIIB association with TFIIIC2 and pol III in solution is reduced, as is the TFIIIB association with tRNA promoters in vivo (Crighton et al. 2003). Our results suggest that at least in certain cell types such as IMR90 cells, pol III transcription repression results, in addition, from an inhibition of the enzyme itself. Thus, MMS treatment apparently inactivates several components of the RNA polymerase III transcription machinery, and at least in the case of the enzyme itself, the extent of this inactivation varies in different cell types. Whether inactivation of RNA polymerase III is, like that of TFIIIB, dependent on p53, remains to be determined.

We have shown before that CK2 phosphorylation of the pol III complex is required for U6 transcription (Hu et al. 2003). MMS treatment might result in dephosphorylation and thus inactivation of the pol III complex. How- 
ever, incubation of the pol III complex from MMStreated cells with CK2 followed by a CK2 inhibitor, thus allowing phosphorylation (Fig. 4D, lanes labeled CK2; see also Hu et al. 2003), did not restore transcription (Fig. $4 \mathrm{D}$, lanes 4,5), suggesting that CK2 phosphorylation is not the only requirement to activate pol III from MMStreated IMR90 cells.

Since upon MMS treatment we observed dissociation of $\beta$-actin from the pol III complex (Fig. 3C), we then wondered whether pol III from MMS-treated cells might be inactive because of the lack of $\beta$-actin. We therefore added to the minimal system recombinant, Escherichia coli expressed $\beta$-actin carrying an $\mathrm{N}$-terminal Flag tag and a C-terminal His tag, and tested the ability of pol III from MMS-treated cells to support pol III transcription when treated either first with a CK2 inhibitor and then with CK2, thus preventing CK2 phosphorylation (Fig. 4D, lanes labeled $+\mathrm{I}$ ), or first with CK2 and then with a CK2 inhibitor, thus allowing phosphorylation. Strikingly, in the presence of $\beta$-actin, CK2-treated pol III from MMS-treated cells was capable of supporting U6 transcription in the minimal system (Fig. 4D, cf. lanes 8,9 and 4-7). Thus, inactive pol III purified from MMStreated IMR90 cells can be activated by a combination of treatment with CK2 and addition of $\beta$-actin. This result has several implications. First, the target for CK2 phosphorylation within the pol III complex has not been defined. Since a pol III complex devoid of $\beta$-actin needs to be phosphorylated by CK2 to regain activity, the CK2 target in the pol III complex is unlikely to be $\beta$-actin. Instead, it may correspond to one of the pol III subunits or to one of the other tightly associated polypeptides found in the preparation (see $\mathrm{Hu}$ et al. 2003). Second, the requirement for addition of $\beta$-actin suggests that $\beta$-actin itself is required for pol III transcription. Most of the nuclear actin is thought to be in a form different from its cytoplasmic filamentous form, perhaps in a monomeric form (Bettinger et al. 2004). Moreover, since fusion of the VP16 activation domain to the $\mathrm{C}$ terminus of actin prevents polymerization (Posern et al. 2002), it is possible that the recombinant actin we used to reconstitute transcription, which carried small tags at both the $\mathrm{N}$ and $\mathrm{C}$ termini, may not have been able to polymerize. Thus, although this is an open question, it is conceivable that the form of actin required for pol III transcription is monomeric actin.

Although several reports indicate that actin may be required for transcription in vivo, there is, so far, little evidence that it is required for the transcription process itself rather than for proper localization or configuration of transcription units. The observation that $\beta$-actin is required for pol III transcription in a purified system suggests a direct role in basal transcription. What this role consists of is presently not known, but it may not be limited to just pol III transcription. Indeed, a number of years ago, an activity that stimulated pol II transcription in an in vitro transcription system was found to copurify with actin, raising the possibility that actin is a pol II transcription factor (Egly et al. 1984), and more recently, actin was shown to be associated with pol I (Fomproix and Percipalle 2004). It is conceivable, then, that actin is required for transcription by all three RNA polymerases.

\section{Materials and methods}

The purification of pol III from untagged cells was performed as described by Sepehri Chong et al. (2001).
The chromatin immunoprecipitations in Figure 2 were performed as described by Schramm et al. (2000), Zhao et al. (2001), and Hu et al. (2003). The promoter sequence of an actively transcribed U6 gene was amplified with primers U6UP4 and U6L2, which amplify the U6-1 promoter region. The control primers (U64-1 and U64-120) amplified the promoter region of the U6-4 gene, which is transcriptionally inactive (Domitrovich and Kunkel 2003). The chromatin immunoprecipitation in Figure 4A was performed with the same primers as in Figure 2, but quantitative PCR (qPCR) was used for amplification (Hu et al. 2004). The double-stranded amplicons were labeled with SYBR, and the fluorescent signals were detected with an ABI Prism 770 sequence-detection system (Applied Biosystems). The qPCR conditions were one cycle with a 2-min incubation at $50^{\circ} \mathrm{C}$, followed by a 10 -min incubation at $95^{\circ} \mathrm{C}$, followed by 40 cycles with a 15 -sec incubation at $95^{\circ} \mathrm{C}$ and 1 -min incubation at $60^{\circ} \mathrm{C}$.

For the images in Figure 3D, IMR90 cells were grown on coverslips overnight at $37^{\circ} \mathrm{C}$ and then treated for $2 \mathrm{~h}$ with either $1 \mathrm{mM}$ MMS in DMSO or with DMSO alone. For the anti- $\alpha$-tubulin staining, the cells were then washed three times with prewarmed PBS, fixed with $2 \%$ paraformaldehyde for $15 \mathrm{~min}$ at room temperature, washed three times with PBS, and permeabilized with $0.5 \%$ Triton X-100 for 5 min at room temperature. The cells were then washed three times with PBS, incubated with $0.5 \%$ goat serum in PBS for $1 \mathrm{~h}$, and with an anti- $\alpha$-tubulin antibody (1:1000, Sigma) for $1 \mathrm{~h}$ at room temperature. The cells were washed three times with PBS followed by a 1-h incubation with Alexa Fluor 488-conjugated goat anti-mouse IgG (Molecular Probes) at a 1:1000 dilution in $0.5 \%$ goat serum. The slides were then washed four times with PBS. For the actin stain, the cells were processed as above except that fixation was with $3.7 \%$ formaldehyde for $10 \mathrm{~min}$ at room temperature, and the cells were stained with Alexa Fluor 568-labeled phalloidin (1:40, Molecular Probe) in the presence of $1 \%$ BSA at room temperature for $20 \mathrm{~min}$. The DNA was visualized by staining with 4', 6'-diamidino-2-phenylindole (DAPI) for $5 \mathrm{~min}$ at room temperature. The images were collected with a Zeiss fluorescent microscope.

In Figure 4D, pol III was immunoprecipitated from either actively growing or MMS-treated IMR90 cells with anti-RPC4 antibody beads. The beads were washed three times with a buffer containing $400 \mathrm{mM}$ $\mathrm{KCl}$ and once with a buffer containing $100 \mathrm{mM} \mathrm{KCl}$. The bound material was eluted in Laemmli buffer and analyzed by immunoblotting with anti-RPC1. The enzyme was complemented with $35 \mathrm{ng}$ of TBP, $60 \mathrm{ng}$ of Brf2, $130 \mathrm{ng}$ of Bdp1, $550 \mathrm{ng}$ of $\mathrm{SNAP}_{\mathrm{c}}$, and 50 and $100 \mathrm{ng}$ of recombinant $\beta$-actin. Recombinant $\beta$-actin was expressed in E. coli and carried an $\mathrm{N}$-terminal Flag tag and a C-terminal His tag.

\section{Acknowledgments}

We thank Y. Sun for technical assistance and J. Duffy and P. Renna for artwork and photography. This work was funded in part by NIH grant GM38810. N.H. is supported by the Howard Hughes Medical Institute.

\section{References}

Ascough, K.R. 2004. Endocytosis: Actin in the driving seat. Curr. Biol. 14: R124-R126.

Bartholomew, B., Durkovich, D., Kassavetis, G.A., and Geiduschek, E.P. 1993. Orientation and topography of RNA polymerase III in transcription complexes. Mol. Cell. Biol. 13: 942-952.

Bettinger, B.T., Gilbert, D.M., and Amberg, D.C. 2004. Actin up in the nucleus. Nat. Rev. Mol. Cell. Biol. 5: 410-415.

Brakebusch, C. and Fassler, R. 2003. The integrin-actin connection, an eternal love affair. EMBO J. 22: 2324-2333.

Cramer, P., Bushnell, D.A., and Kornberg, R.D. 2001. Structural basis of transcription: RNA polymerase II at 2.8 angstrom resolution. Science 292: $1863-1876$.

Crighton, D., Woiwode, A., Zhang, C., Mandavia, N., Morton, J.P., Warnock, L.J., Milner, J., White, R.J., and Johnson, D.L. 2003. p53 represses RNA polymerase III transcription by targeting TBP and inhibiting promoter occupancy by TFIIIB. EMBO J. 22: 2810-2820.

Domitrovich, A.M. and Kunkel, G.R. 2003. Multiple, dispersed human U6 small nuclear RNA genes with varied transcriptional efficiencies. Nucleic Acids Res. 31: 2344-2352.

Egly, J.M., Miyamoto, N.G., Moncollin, V., and Chambon, P. 1984. Is actin a transcription initiation factor for RNA polymerase B? EMBO J. 3: $2363-2371$. 
Engqvist-Goldstein, A.E. and Drubin, D.G. 2003. Actin assembly and endocytosis: From yeast to mammals. Annu. Rev. Cell Dev. Biol. 19: 287-332.

Fomproix, N. and Percipalle, P. 2004. An actin-myosin complex on actively transcribing genes. Exp. Cell Res. 294: 140-148.

Hu, P., Wu, S., Sun, Y., Yuan, C.C., Kobayashi, R., Myers, M.P., and Hernandez, N. 2002. Characterization of human RNA polymerase III identifies orthologues for Saccharomyces cerevisiae RNA polymerase III subunits. Mol. Cell. Biol. 22: 8044-8055.

$\mathrm{Hu}, \mathrm{P} ., \mathrm{Wu}, \mathrm{S}$., and Hernandez, N. 2003. A minimal RNA polymerase III transcription system from human cells reveals positive and negative regulatory roles for CK2. Mol. Cell 12: 699-709.

Hu, P., Samudre, K., Wu, S., Sun, Y., and Hernandez, N. 2004. CK2 phosphorylation of Bdpl executes cell cycle-specific RNA polymerase III transcription repression. Mol. Cell 16: 81-92.

Percipalle, P., Fomproix, N., Kylberg, K., Miralles, F., Bjorkroth, B., Daneholt, B., and Visa, N. 2003. An actin-ribonucleoprotein interaction is involved in transcription by RNA polymerase II. Proc. Natl. Acad. Sci. 100: 6475-6480.

Posern, G., Sotiropoulos, A., and Treisman, R. 2002. Mutant actins demonstrate a role for unpolymerized actin in control of transcription by serum response factor. Mol. Biol. Cell 13: 4167-4178.

Rando, O.J., Zhao, K., Janmey, P., and Crabtree, G.R. 2002. Phosphatidylinositol-dependent actin filament binding by the SWI/SNF-like BAF chromatin remodeling complex. Proc. Natl. Acad. Sci. 99: 28242829

Scheer, U., Hinssen, H., Franke, W.W., and Jockusch, B.M. 1984. Microinjection of actin-binding proteins and actin antibodies demonstrates involvement of nuclear actin in transcription of lampbrush chromosomes. Cell 39: 111-122.

Schramm, L. and Hernandez, N. 2002. Recruitment of RNA polymerase III to its target promoters. Genes \& Dev. 16: 2593-2620.

Schramm, L., Pendergrast, P.S., Sun, Y., and Hernandez, N. 2000. Different human TFIIIB activities direct RNA polymerase III transcription from TATA-containing and TATA-less promoters. Genes \& Dev. 14: 2650-2663.

Sepehri Chong, S., Hu, P., and Hernandez. N. 2001. Reconstitution of transcription from the human U6 small nuclear RNA promoter with eight recombinant polypeptides and a partially purified RNA polymerase III complex. J. Biol. Chem. 276: 20727-20734.

Shen, X., Ranallo, R., Choi, E., and Wu, C. 2003. Involvement of actinrelated proteins in ATP-dependent chromatin remodeling. Mol. Cell 12: $147-155$.

Suetsugu, S. and Takenawa, T. 2003. Regulation of cortical actin networks in cell migration. Int. Rev. Cytol. 229: 245-286.

Wang, Z. and Roeder, R. 1997. Three human RNA polymerase III-specific subunits form a subcomplex with a selective function in specific transcription initiation. Genes \& Dev. 11: 1315-1326.

Zhao, K., Wang, W., Rando, O.J., Xue, Y., Swiderek, K., Kuo, A., and Crabtree, G.R. 1998. Rapid and phosphoinositol-dependent binding of the SWI/SNF-like BAF complex to chromatin after T lymphocyte receptor signaling. Cell 95: 625-636.

Zhao, X., Pendergrast, P.S., and Hernandez, N. 2001. A positioned nucleosome on the human U6 promoter allows recruitment of SNAP $_{\mathrm{c}}$ by the Oct-1 POU domain. Mol. Cell 7: 539-549. 


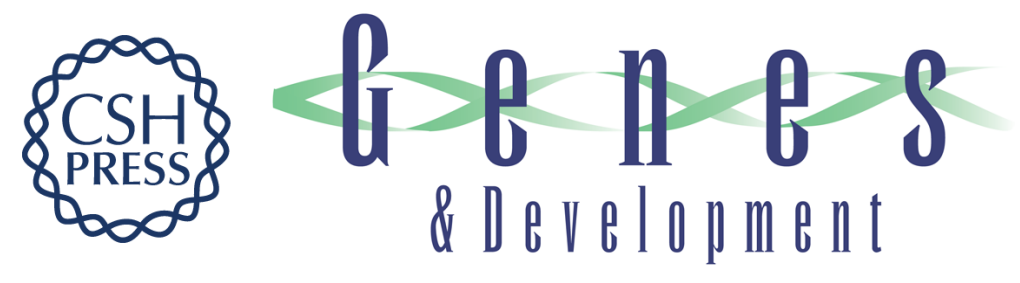

\section{A role for $\beta$-actin in RNA polymerase III transcription}

Ping Hu, Si Wu and Nouria Hernandez

Genes Dev. 2004, 18:

Access the most recent version at doi:10.1101/gad.1250804

Supplemental
Material http://genesdev.cshlp.org/content/suppl/2004/12/08/gad.1250804.DC1

References This article cites 25 articles, 12 of which can be accessed free at: http://genesdev.cshlp.org/content/18/24/3010.full.html\#ref-list-1

License

Email Alerting Receive free email alerts when new articles cite this article - sign up in the box at the top Service right corner of the article or click here.

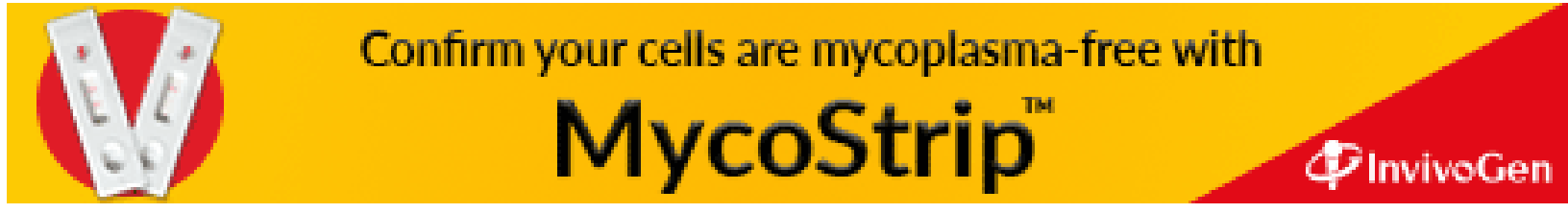

